\title{
Nintedanib selectively inhibits the activation and tumour-promoting effects of fibroblasts from lung adenocarcinoma patients
}

\author{
M Gabasa ${ }^{1}$, R Ikemori $^{1}$, F Hilberg ${ }^{2}$, N Reguart ${ }^{\star, 3}$ and J Alcaraz ${ }^{\star, 1,4}$ \\ ${ }^{1}$ Unit of Biophysics and Bioengineering, Department of Biomedicine, School of Medicine and Health Sciences, Universitat de \\ Barcelona, Barcelona 08036, Spain; ${ }^{2}$ Boehringer Ingelheim Austria RCV GmbH \& Co KG, Vienna 1120, Austria; ${ }^{3}$ Medical Oncology \\ Department, Hospital Clínic de Barcelona, Institut d'Investigacions Biomèdiques August Pi i Sunyer (IDIBAPS), Barcelona 08036, \\ Spain and ${ }^{4}$ CIBER de Enfermedades Respiratorias, Madrid 28029, Spain
}

Background: Nintedanib is a clinically approved multikinase receptor inhibitor to treat non-small cell lung cancer with adenocarcinoma (ADC) histology in combination with docetaxel, based on the clinical benefits reported on ADC but not on squamous cell carcinoma (SCC), which are the two most common histologic lung cancer subtypes.

Methods: We examined the potential role of tumour-associated fibroblasts (TAFs) in the differential effects of nintedanib in ADC and SCC. Because TAFs are largely quiescent and activated in histologic sections, we focused on the antifibrotic effects of nintedanib on TAFs stimulated with the potent fibroblast activator TGF- $\beta 1$, which is upregulated in lung cancer.

Results: Nintedanib dose-dependently inhibited the TGF- $\beta 1$-induced expression of a panel of pro-fibrotic activation markers in both ADC-TAFs and control fibroblasts derived from uninvolved lung parenchyma, whereas such inhibition was very modest in SCC-TAFs. Remarkably, nintedanib abrogated the stimulation of growth and invasion in a panel of carcinoma cell lines induced by secreted factors from activated TAFs in ADC but not SCC, thereby supporting that TGF- $\beta$ signalling and aberrant TAF-carcinoma cross-talk is regulated by different mechanisms in ADC and SCC.

Conclusions: These results reveal that nintedanib is an effective inhibitor of fibrosis and its associated tumour-promoting effects in ADC, and that the poor antifibrotic response of SCC-TAFs to nintedanib may contribute to the differential clinical benefit observed in both subtypes. Our findings also support that preclinical models based on carcinoma-TAF interactions may help defining the mechanisms of the poor antifibrotic response of SCC-TAFs to nintedanib and testing new combined therapies to further expand the therapeutic effects of this drug in solid tumours.

Lung cancer is the leading cause of cancer-related deaths in both men and women in industrialised countries, with a 5-year survival rate of only $\sim 15 \%$. Histologically, $\sim 85 \%$ of lung cancer patients are classified as non-small cell lung cancer (NSCLC), which are further subdivided into adenocarcinoma (ADC; 50\%), squamous cell carcinoma (SCC; $\sim 40 \%$ ) and other less frequent subtypes (Ferlay et al, 2013; Chen et al, 2014). Even though both ADC and SCC are epithelial in origin, it is increasingly acknowledged that carcinoma cells need to surround themselves by an aberrant stiff desmoplastic stroma to develop a tumour (Hanahan and Weinberg, 2011; Bissell and Hines, 2012). Likewise, a large body of work indicates that tumour-associated fibroblasts (TAFs), which are the most abundant stromal cell type, are implicated in many critical steps of cancer progression including initiation, growth, invasion, metastasis, stemness, immunosuppression and even resistance to therapies (Kalluri and Zeisberg, 2006; Ohlund et al, 2014; Lugo et al, 2016; Labernadie et al, 2017). Accordingly, targeting the aberrant carcinoma-TAF interactions is emerging as 
a promising therapeutic strategy in NSCLC and other solid tumours (Ohlund et al, 2014; Lugo et al, 2016).

Nintedanib is a small molecule inhibitor of multiple tyrosine kinases that has been recently approved for second-line treatment after chemotherapy failure combined with the cytotoxic docetaxel in patients with advanced lung ADC, owing to the selective positive therapeutic results reported in ADC (but not in SCC) patients in a phase III clinical trial (LUME-Lung 1) (Reck et al, 2014). Nintedanib (also known as BIBF 1120) competitively binds to the ATP-binding sites within the kinase domains of VEGFR receptors (VEGFR) $1-3$, PDGFR $\alpha / \beta$, and FGFR 1-4. In addition, nintedanib inhibits Src family tyrosine kinases (Src, Lck, Lyn), Flt-3 and RET (Hilberg et al, 2008; Wollin et al, 2014; Awasthi and Schwarz, 2015). In agreement with its initial design as a next generation antiangiogenic drug, previous studies with lung cancer xenografts reported that nintedanib exhibits strong antiangiogenic functions through a direct effect on cell types involved in angiogenesis, including endothelial cells, pericytes and smooth muscle cells (Hilberg et al, 2008). In addition, it was reported that nintedanib exhibits further anticancer effects by reducing tumour growth and metastasis (Hilberg et al, 2008; Cenik et al, 2013). These previous observations highlight the ability of nintedanib to target multiple cell types within the tumour microenvironment, which has been associated with the simultaneous inhibitory effects of this drug on several signalling pathways (Wollin et al, 2014; Huang et al, 2016). However, the biological processes underlying both the positive therapeutic effects of nintedanib in ADC and the lack of such effects in SCC remain unknown.

In addition to lung ADC, nintedanib has been recently approved to treat patients with idiopathic pulmonary fibrosis (IPF), based on its ability to slow the progression of this rare disease in clinical trials (Richeldi et al, 2014; Wollin et al, 2015). Although lung ADC and IPF are markedly distinct both clinically and biologically, pulmonary tissues in both diseases have in common an abundant stiff desmoplastic stroma rich in collagen and activated fibroblasts/ myofibroblasts (King et al, 2011). Because IPF-progression has been strongly associated with the persistent proliferation and pathologic activation of lung fibroblasts (King et al, 2011; Wollin et al, 2015), analysing the effects of nintedanib on this cell type has been the subject of increasing preclinical research. In vitro studies have shown that nintedanib interferes with important fibroblastdependent hallmarks of lung fibrosis including fibroblast proliferation, activation and deposition of extracellular matrix components in response to pro-fibrotic cytokines like PDGF or TGF- $\beta$ (Wollin et al, 2015). These studies clearly indicate that nintedanib has a direct impact on fibroblast functions, thereby suggesting that this drug may also have direct inhibitory effects on TAFs. However, to our knowledge, no studies have been undertaken to examine the impact of nintedanib on activated TAFs.

We have recently shown that ADC-TAFs and SCC-TAFs are activated in vivo and overexpress activation markers compared to paired control fibroblasts in vitro. Yet ADC-TAFs and SCC-TAFs exhibited also distinct phenotypic alterations in terms of growth regulation and integrin signalling (Puig et al, 2015; Lugo et al, 2016). Therefore, given the known direct effects of nintedanib on lung fibroblasts and the distinct aberrant phenotypes of ADCTAFs and SCC-TAFs, we hypothesised that the selective therapeutic effects of nintedanib on ADC may be due, at least in part, to a larger impact of this drug on ADC-TAFs compared to SCC-TAFs. To test this hypothesis, we examined the inhibitory effects of nintedanib in lung TAFs from ADC and SCC patients as well as lung fibroblast from non-malignant tissue upon stimulation with either growth factors or TGF- $\beta 1$ in terms of growth and activation, respectively. Moreover we examined the potential inhibitory role of nintedanib on the tumour-promoting effects of activated TAFs in terms of growth and invasion in a panel of ADC and SCC cell lines.

\section{MATERIALS AND METHODS}

Tissue samples and primary human lung TAFs. Lung tissue samples were obtained from a cohort of 20 early stage NSCLC surgical patients (10 ADC, 10 SCC) prior to 2013 at the Hospital Clinic de Barcelona (HCB, Spain) with the approval of the Ethics Committees of the HCB and the Universitat de Barcelona. All patients gave their informed consent. Selected patients were male, chemo-naïve, Caucasian, $\geqslant 55$ years old, and current smokers with confirmed ADC or SCC diagnosis (additional clinical characteristics of patients are available elsewhere (Vizoso et al, 2015)). Tumour samples were collected and divided into two parts. One part was paraffin-embedded for histologic analysis and the remaining was used to isolate TAFs by outgrowth of tissue explants (Puig et al, 2015). The mesenchymal origin of the primary fibroblasts was confirmed by their positive and negative immunofluorescence staining with vimentin and pan-cytokeratin antibodies, respectively (Vizoso et al, 2015).

Histology. Immunohistochemical analyses of Ki-67 and $\alpha$-SMA were performed on tissue sections from our cohort using the Bond automated immunohistochemistry system (Leica Microsystems, Wetzlar, Germany) as previously described (Puig et al, 2015; Vizoso et al, 2015; Lugo et al, 2016). Primary antibodies included anti- $\alpha$-SMA (1A4) and anti-Ki-67 (MIB-1, Dako, Cambridge, UK). Nuclei were counterstained with haematoxylin. Histological stainings were imaged with a bright-field microscope (BX43) coupled to a digital camera (DP72) using a $\times 40$ objective (Olympus, Japan). All image processing was carried out with ImageJ (Abramoff et al, 2004).

Cell culture. The human fibroblast cell line CCD-19Lu obtained from normal pulmonary tissue (ATCC, USA), patient-derived primary lung TAFs and paired fibroblasts from uninvolved pulmonary tissue were maintained in DMEM-based fibroblast culture medium as described elsewhere (Bertolini et al, 2015; Puig et al, 2015). Data from paired fibroblasts and CCD-19Lu were pooled and used as control fibroblasts based on previous results from our group (Puig et al, 2015). Fibroblasts were used up to passage 10 (CCD-19Lu) or 6 (primary fibroblasts) to avoid replicative senescence (Navab et al, 2011). All experiments with fibroblasts were carried out on tissue culture plastic substrata coated with $0.1 \mathrm{mg} \mathrm{ml}^{-1}$ collagen-I solution (Millipore, Billerica, MA, USA) overnight at $4{ }^{\circ} \mathrm{C}$. Unless otherwise indicated, fibroblasts were seeded at $8 \times 10^{3}$ cells $\mathrm{cm}^{-2}$. To induce either proliferation or activation, fibroblasts were stimulated with $10 \%$ FBS (Gibco, Waltham, MA, USA) or $2.5 \mathrm{ng} \mathrm{ml}^{-1}$ human recombinant human TGF- $\beta 1$ (R\&D Systems, Minneapolis, MN, USA), respectively, concomitantly with increasing doses of nintedanib. Nintedanib (BIBF 1120) was provided by Boehringer Ingelheim Pharma GmbH \& Co (Vienna, Austria), was diluted in DMSO and used at concentrations $0.5-5 \mu \mathrm{M}$ based on previous studies (Hilberg et al, 2008; Wollin et al, 2014; Rangarajan et al, 2016; Shochet et al, 2016). For flow cytometry experiments, fibroblasts were seeded in fibroblast culture medium for $24 \mathrm{~h}$ and maintained in either $0 \%$ FBS (serum-free) or $10 \%$ FBS medium in the presence of increasing nintedanib doses for 5 days with a media change at day 3.

A panel of lung carcinoma cell lines derived from either ADC (H1437, H522) or SCC (SK-MES-1, H520) (ATCC) patients were used to examine the tumour-promoting effects of TAFs treated with nintedanib. All carcinoma cell lines were wild-type EGFR and KRAS (Blanco et al, 2009) to mimic critical genetic aspects of lung ADC patients that may be treated with nintedanib in the clinic (Bronte et al, 2016). Cancer cells were maintained in RPMI-1640-based medium supplemented with $10 \%$ of FBS as reported elsewhere (referred to as epithelial culture medium) (Lugo et al, 2016). 
Conditioned medium. Fibroblasts were seeded as $8 \times 10^{3}$ cells $\mathrm{cm}^{-2}$ in $60 \mathrm{~cm}^{2}$ Petri dishes in serum-free fibroblast medium supplemented with $2.5 \mathrm{ng} \mathrm{ml}^{-1}$ TGF- $\beta 1$ with our without $2 \mu \mathrm{M}$ nintedanib for 3 days. Next, culture medium was washed and replaced with nintedanib-free and serum-free fibroblast culture medium for $48 \mathrm{~h}$. Afterwards, the conditioned medium (CM) was collected, centrifuged to remove suspended cells and stored at $-20{ }^{\circ} \mathrm{C}$ until use.

Fibroblast cell number density and viability. The number density of viable fibroblasts was assessed with the trypan blue exclusion assay using a hemocytometer (Mesner et al, 1992). The percentage of viable fibroblasts was assessed as the ratio of viable cell number density with respect to total cell number density.

Cell cycle analysis. DNA histograms of single fibroblasts were obtained by flow cytometry (LSRFortessa, BD Biosciences, San Jose, CA, USA), and the percentages of cycling fibroblasts (i.e., cells in S or G2/M phases) were assessed with FACSDiva Software v6.1.3 (BD Biosciences) as described elsewhere (Puig et al, 2015; Lugo et al, 2016).

Quantitative PCR with reverse transcription. RNA extraction and reverse transcription was conducted as previously reported by our group (Vizoso et al, 2015). Real-time PCR reactions were performed on $20 \mathrm{ng}$ of each cDNA sample using TaqMan Gene Expression Master Mix and TaqMan gene-specific primer pairs and probes (Applied Biosystems, USA) for COL1A1, COL3A1 and $P O L R 2 A$ (used as a housekeeping gene). Relative gene expression with respect to the housekeeping gene was assessed as $2^{-\Delta \mathrm{Ct}}$ (Livak and Schmittgen, 2001).

Western blotting. Fibroblasts were lysed in extraction buffer supplemented with phosphatase and protease inhibitors as described before (Puig et al, 2015). Equal protein amounts were separated on a $10 \%$ Mini-PROTEAN TGX precast gels (Bio-Rad, Hercules, CA, USA), transferred to a PVDF membrane (GE Healthcare, Little Chalfont, UK), blocked and incubated overnight with suitable primary antibodies including anti- $\alpha$-SMA (Sigma, St Louis, MO, USA), P4HA2 (Proteintech Europe, Manchester, UK) or $\beta$-actin (Sigma, used as loading control). Protein bands were labelled and visualised by chemiluminescence in a digital imaging system (ImageQuant LAS 4000, GE Healthcare). Protein band intensities were quantified with ImageJ and normalised to the corresponding loading control.

Assessment of $I C_{50}$ values describing the antiproliferative and antifibrotic effects of nintedanib in lung fibroblasts. Selected quantitative assays (fold cell number, COL1A1 and COL3A1 expression) were used to compute $I C_{50}$ values indicative of the antiproliferative and antifibrotic effects of nintedanib, respectively. For this purpose, the data corresponding to the measurements from each assay $(y)$ at different nintedanib concentrations $(x)$ were fitted to an exponential decay function $\left(y=y_{o}+a \mathrm{e}^{-b \cdot x}\right.$, where $y_{o}$, $a$ and $b$ were the fitting parameters) with SigmaPlot (Systat Software, USA), and used to assess $I C_{50}=-\ln 0.5 / b$. The error $\sigma_{I C 50}$ associated with the calculation of $I C_{50}$ was assessed using error propagation (Taylor, 1997) as the absolute value of $\sigma_{I C 50}=$ $\sigma_{b} \cdot \ln 0.5 / b^{2}$, where $\sigma_{b}$ is the uncertainty of the $b$ fitting.

Carcinoma cell growth stimulated by CM from TAFs. Carcinoma cell growth stimulated by CM from activated TAFs was assessed using protocols previously described by our group (Lugo et al, 2016). Briefly, for each cancer cell line, cancer cells were plated at $1.1 \times 10^{4}$ cells $\mathrm{cm}^{-2}$ in six-well plates and grown in $2 \mathrm{ml}$ of a $1: 1$ mixture of CM and serum-free epithelial culture medium for 3 days. A 1:1 mixture of serum-free fibroblast/epithelial culture medium was used as (basal) control medium. Afterwards, cells were fixed with $4 \%$ PFA and their nuclei counterstained with Hoechst 33342 (Molecular Probes, Eugene, OR, USA). Fluorescent nuclei were imaged and counted with ImageJ. Cell number density was assessed as the average nuclear density/image.

Carcinoma cell invasion stimulated by CM from TAFs. Cancer cells were seeded on Matrigel-coated filter-inserts as $15 \times 10^{4}$ cells $\mathrm{cm}^{-2}$ in serum-free epithelial culture medium as described (Lugo et al, 2016). CM or control medium was added to the lower Transwell compartment. After $48 \mathrm{~h}$, cells were fixed with $100 \%$ methanol and incubated with $0.5 \%$ crystal violet (Sigma). Cells that had invaded into the lower side of the filter were imaged by a phase-contrast microscope provided with a colour camera. Invasion was computed as the average crystal violet positive area/image. All image processing was conducted with ImageJ.

Statistical analysis. Two group comparisons were performed with Student's $t$-test (SigmaPlot). Statistical significance was assumed at $P<0.05$. All experiments were conducted as triplicates. All data shown are mean \pm s.e.

\section{RESULTS}

Nintedanib inhibits both growth and activation of control lung fibroblasts. To mimic either a pro-growth or a pro-fibrotic stiff microenvironment in vitro, we stimulated fibroblasts cultured on collagen-coated tissue culture plastic with either 10\% FBS (which is rich in the fibroblast mitogen PDGF (Freshney, 1994)) or $2.5 \mathrm{ng} \mathrm{ml}^{-1}$ of the potent fibroblast activator TGF- $\beta 1$ (Hinz et al, 2012), respectively. As expected, such stimulations induced a robust increase in either fibroblast proliferation (10\% FBS) or activation (TGF- $\beta 1$ ) (Supplementary Figure S1). To identify a suitable dose range of nintedanib, we first examined the impact of this drug on the viability and growth of control fibroblasts (Figure 1). Nintedanib did not compromise the viability of fibroblasts cultured in $10 \%$ FBS for 5 days up to $5 \mu \mathrm{M}$ (Figure 1A). In contrast, nintedanib prevented the increase in fibroblast population upon mitogenic stimulation with 10\% FBS in a dose-dependent manner (Figure 1B), whereas DMSO vehicle did not (Supplementary Figure S1). To confirm the antiproliferative effects of nintedanib, we analysed the percentage of cycling fibroblasts by flow cytometry. In qualitative agreement with our fibroblast number density data, nintedanib downregulated the rise in cycling fibroblasts upon stimulation with 10\% FBS for 5 days (Figure 1C). A marked drop in cycling fibroblasts was also observed after only $12 \mathrm{~h}$ upon $2 \mu \mathrm{m}$ nintedanib treatment, indicating that this drug induces an early growth arrest in fibroblasts (Figure 1D).

We next examined the antifibrotic effects of nintedanib by assessing the expression of a panel of pro-fibrotic markers including alpha-smooth muscle actin ( $\alpha$-SMA), fibrillar collagens (COL1A1 and COL3A1) and prolyl-4-hydroxylase $\alpha$ subunit 2 (P4HA2) - an essential enzyme for collagen biosynthesis that is upregulated by TGF- $\beta$ (Koslowski et al, 2003; Kalluri and Zeisberg, 2006) - by either western blotting or qRT-PCR (Kalluri and Zeisberg, 2006; Hinz et al, 2012; Ohlund et al, 2014) in control fibroblasts stimulated with TGF- $\beta 1$ or DMSO vehicle for 3 days. Nintedanib downregulated the marked induction of both COL1A1 and $\alpha$-SMA upon concomitant stimulation with TGF- $\beta 1$ in a dosedependent fashion (Figure 1E and F), whereas DMSO vehicle did not (Supplementary Figure S1). Likewise nintedanib dose-dependently reduced both COL $3 A 1$ and $\mathrm{P} 4 \mathrm{HA} 2$ expression in control fibroblasts (Figure $1 \mathrm{G}$ and $\mathrm{H}$ ). Remarkably nintedanib even reduced COL1A1 and $\alpha$-SMA in control fibroblasts pre-activated with TGF- $\beta 1$ for 3 days (Figure 1I and J). These results underscore the robust inhibitory effects of this drug on the expression of both fibrillar collagens and collagen-related genes. Altogether our results expand previous observations of the antiproliferative and antifibrotic effects of nintedanib in normal and IPF-derived lung 
A

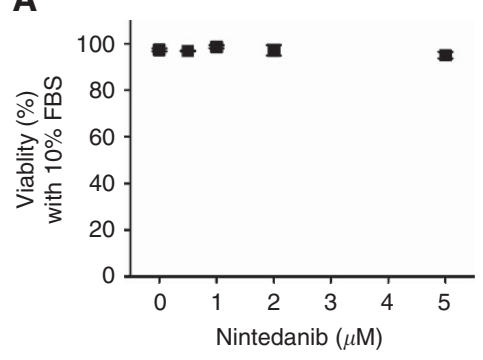

C

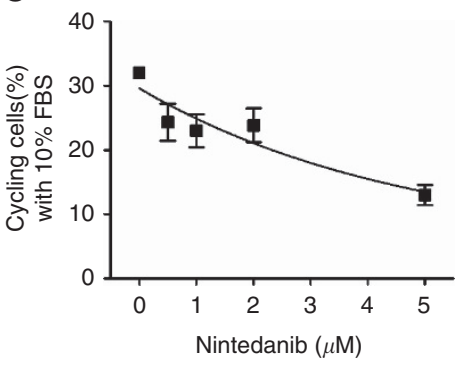

B
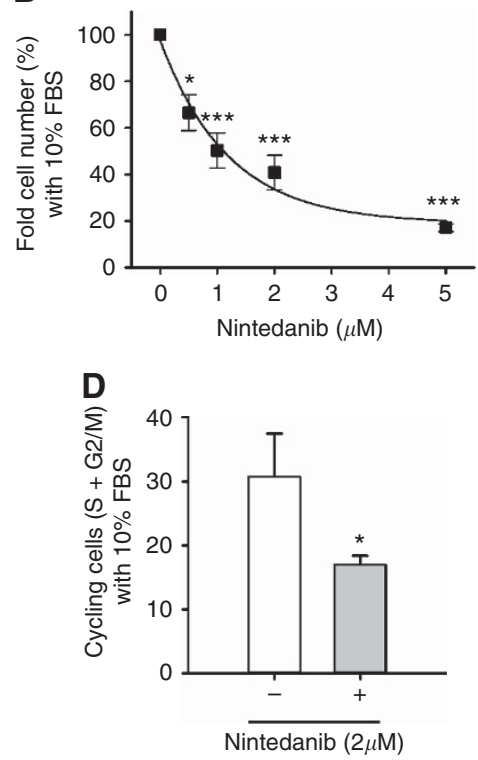

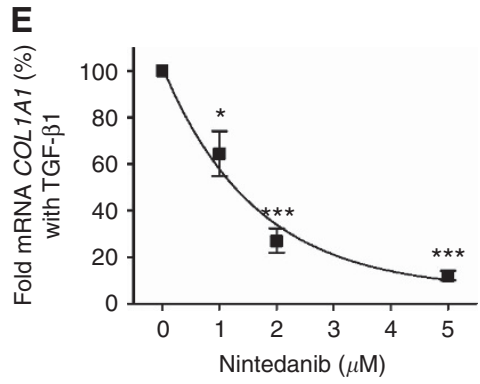

G
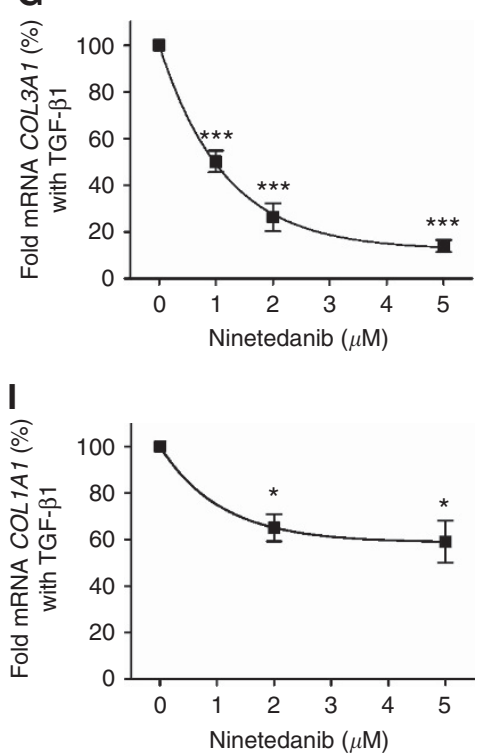

F
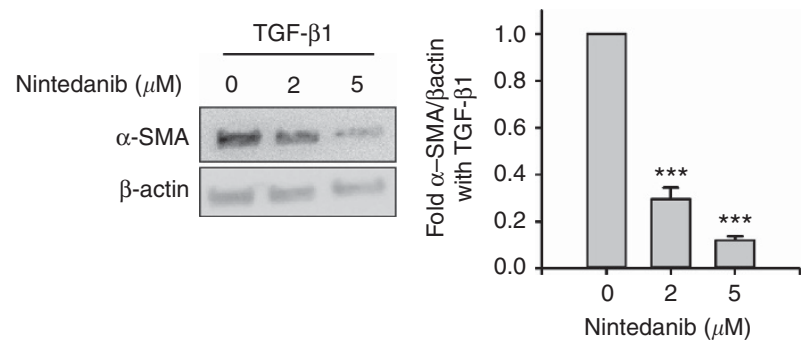

H
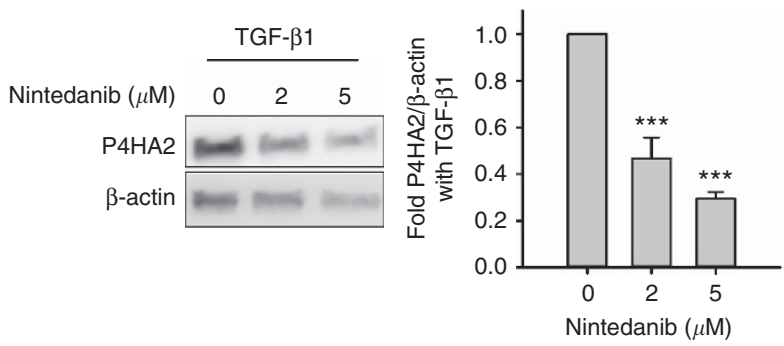

J

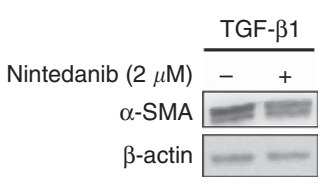

Figure 1. Antiproliferative and antifibrotic effects of nintedanib in control lung fibroblasts. (A) Percentage of viable fibroblasts cultured with $10 \%$ FBS for 5 days with increasing concentrations of nintedanib $(n=3)$. ( $\mathbf{B}$ and $\mathbf{C})$ Fold cell number $(\mathbf{B}, n=5)$ and percentage of cycling cells (i.e., cells in $\mathrm{S}$ or $\mathrm{G} 2 / \mathrm{M})(\mathbf{C}, n=4)$ of fibroblasts cultured as in $\mathbf{A}$. Continuous line in $\mathbf{B}$ corresponds to the fitting of an exponential decay function that was used to assess $I C_{50}$ shown in Table 1. (D) Percentage of cycling fibroblasts cultured for $12 \mathrm{~h}$ with $10 \% \mathrm{FBS}$ in the absence or presence of $2 \mu \mathrm{m}$ nintedanib $(n=3)$. (E) Fold mRNA expression of COL1A1 in fibroblasts stimulated with $2.5 \mathrm{ng} \mathrm{ml}{ }^{-1}$ TGF- $\beta 1$ for 3 days with increasing concentrations of nintedanib ( $n=5$ ). (F) Left, representative western blot analysis of $\alpha$-SMA and $\beta$-actin (loading control) of fibroblasts cultured as in $\mathbf{E}$. Right, densitometry analysis of $\alpha$-SMA expression normalised to $\beta$-actin $(n=5)$. (G and $\mathbf{H})$ Fold mRNA expression of COL3A1 (G) and western blot analysis of P4HA2 (H) in fibroblasts cultured as in $(E)(n=4)$. (I and J) Expression of COL1A 1 and $\alpha$-SMA in fibroblasts pre-activated with $2.5 \mathrm{ng} \mathrm{ml}^{-1} \mathrm{TGF}^{-\beta 1}$ for 3 days and subsequently treated with $2.5 \mathrm{ng} \mathrm{ml}^{-1}$ TGF- $\beta 1$ in the absence or presence of $2 \mu \mathrm{M}$ nintedanib $(n=3)$. Continuous lines in ( $\mathbf{E}, \mathbf{G}$ and $\left.\mathbf{I}\right)$ correspond to fittings of an exponential decay function, which elicited $I C_{50}$ values shown in Table 1 (for $\mathbf{E}$ and $\mathbf{G}$ ) and $0.74 \mu \mathrm{m}$ for $\mathbf{I}$. ${ }^{*} P \leqslant 0.05$, $\star \star P \leqslant 0.01$ and ${ }^{\star \star \star} P \leqslant 0.005$ with respect to $0 \mu \mathrm{m}$ nintedanib were determined by Student's t-test. 
fibroblasts (Hostettler et al, 2014; Wollin et al, 2014; Huang et al, 2016; Rangarajan et al, 2016), and reveal that such inhibitory functions are characterised by $I C_{50} \sim 0.8-1 \mu \mathrm{M}$ (Table 1 ), which is comparable to the maximum nintedanib concentration reported in in vivo studies with this drug (Hilberg et al, 2008).

SCC-TAFs exhibit lower antifibrotic responses to nintedanib than ADC-TAFs, but similar antiproliferative effects. Patientderived lung TAFs overexpress activation markers compared to paired control fibroblasts in both ADC and SCC (Navab et al, 2011; Vizoso et al, 2015; Lugo et al, 2016). Yet we recently reported distinct phenotypic alterations in TAFs from both subtypes, suggesting that they could exhibit subtype-specific responses to nintedanib. To examine this possibility, we first stimulated ADCTAFs and SCC-TAFs with $10 \%$ FBS in the presence of increasing doses of nintedanib or DMSO vehicle. As in control fibroblasts, nintedanib did not compromise the viability of TAFs up to $5 \mu \mathrm{M}$ (Figure 2A). In further agreement with control fibroblasts, nintedanib dose-dependently prevented the increase in both fibroblast number (Figure 2B) and percentage of cycling fibroblasts (Figure 2C) upon 10\% FBS stimulation, whereas DMSO vehicle did not (Supplementary Figure S2). Such marked impact in the cell

Table 1. Nintedanib $I C_{50}(\mu \mathrm{m})$ values characterising the antiproliferative and antifibrotic effects on control fibroblasts and TAFs (mean \pm s.e.)

\begin{tabular}{|l|c|c|c|}
\hline Assay & Control fibroblasts & ADC-TAFs & SCC-TAFs \\
\hline Proliferation & $0.93 \pm 0.09$ & $0.96 \pm 0.09$ & $1.09 \pm 0.05$ \\
\hline COL1A1 & $1.1 \pm 0.3$ & $1.05 \pm 0.07$ & $2.3 \pm 0.6$ \\
\hline COL3A1 & $0.80 \pm 0.03$ & $0.74 \pm 0.15$ & $1.0 \pm 0.1$ \\
\hline
\end{tabular}

Abbreviations: $\mathrm{ADC}=$ adenocarcinoma; $\mathrm{SCC}=$ squamous cell carcinoma; $\mathrm{TAF}=$ tumourassociated fibroblasts. $I C_{50}(\mu \mathrm{M})$ values were computed from Figure $1 \mathrm{~B}, \mathrm{E}$ and $\mathrm{G}$ (control fibroblasts) and Figures $2 \mathrm{~B}$ and $3 \mathrm{~A}$ and $\mathrm{B}$ (TAFs). cycle could be observed at $12 \mathrm{~h}$ upon treatment (Figure 2D), revealing that nintedanib elicits an early growth arrest also in TAFs. These experiments showed that the antiproliferative effects of nintedanib in TAFs were quantitatively similar in both ADC and SCC, eliciting comparable $I C_{50}$ values to each other that were also close to that found in control fibroblasts (Table 1).

Unlike growth inhibition, even though both ADC-TAFs and SCC-TAFs became activated upon TGF- $\beta 1$ stimulation (Supplementary Figure S3), nintedanib inhibition of TAF activation in response to TGF- $\beta 1$ was consistently larger in ADC-TAFs than SCC-TAFs in all the pro-fibrotic markers examined (i.e., fibrillar collagens (Figure 3A and B), $\alpha$-SMA (Figure 3C) and P4HA2 (Figure 3D)) with statistical significance at different nintedanib concentrations, whereas no expression changes were reported upon treatment with DMSO vehicle (Supplementary Figure S2). Such differential antifibrotic responses in TAFs elicited $I C_{50}$ values for COL1A1 and COL3A1 that were 2.2- and 1.4-fold higher in SCC-TAFs than ADC-TAFs, respectively (Table 1). Since $I C_{50}$ values for both fibrillar collagens were very similar in ADCTAFs and control fibroblasts (Table 1), our data reveal for the first time that SCC-TAFs exhibit a poor antifibrotic response to nintedanib, whereas ADC-TAFs exhibit a normal antifibrotic response to this drug.

Lung TAFs are quiescent and activated in vivo, supporting that the subtype-specific antifibrotic function of nintedanib in TAFs may be pathologically relevant. The marked antiproliferative and antifibrotic responses to nintedanib observed in SCC-TAFs and ADC-TAFs in vitro raised the question of what of these effects might be more relevant in vivo. To shed light on this issue, we analysed the proliferative and activation status of TAFs in histologic sections from the same patient cohort used to obtain primary TAFs. Our histologic analysis revealed that TAFs are largely negative for the proliferation marker Ki-67 in both ADC and SCC, whereas a substantial fraction of carcinoma cells were
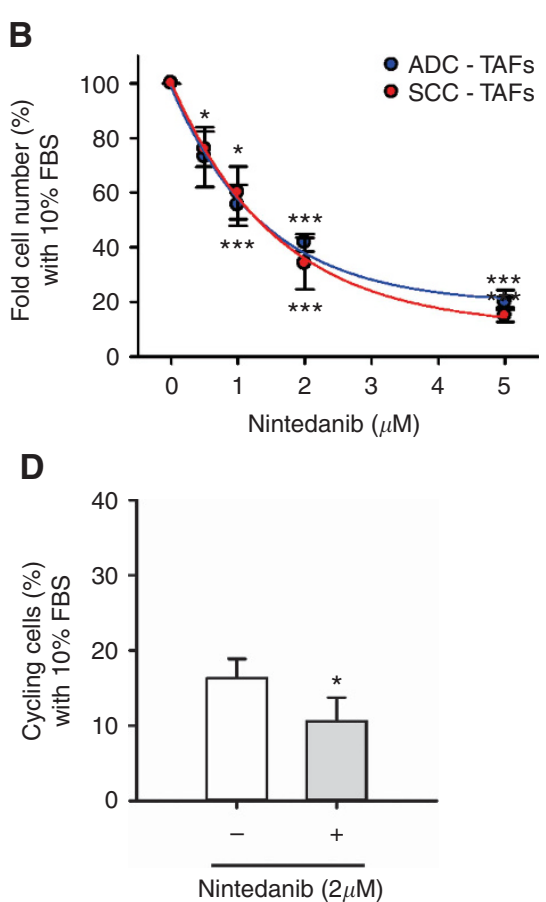

Figure 2. Antiproliferative effects of nintedanib in ADC-TAFs and SCC-TAFs. (A) Viability of TAFs cultured with $10 \%$ FBS for 5 days in the presence of increasing nintedanib concentrations ( $n=3$ ADC, 3 SCC). (B-D) Antiproliferative effects of nintedanib were assessed in terms of fold TAF number ( $\mathbf{B} ; n=3 \mathrm{ADC}, 3 \mathrm{SCC}$ ) and percentage of cycling TAFs ( $; ; n=5 \mathrm{ADC}, 4 \mathrm{SCC}$ ) as in Figure 1. (D) Percentage of cycling TAFs ( $n=2$ ADC, 2 SCC; pooled) after $12 \mathrm{~h}$ in the presence or absence of $2 \mu \mathrm{m}$ nintedanib. Continuous lines in $\mathbf{B}$ and $\mathbf{C}$ correspond to fittings to an exponential decay function, which were used to assess $I C_{50}$ values shown in Table $1 .{ }^{\star} P \leqslant 0.05,{ }^{\star \star} P \leqslant 0.01$ and ${ }^{\star \star \star} P \leqslant 0.005$ with respect to $0 \mu \mathrm{m}$ nintedanib were determined by Student's t-test. 


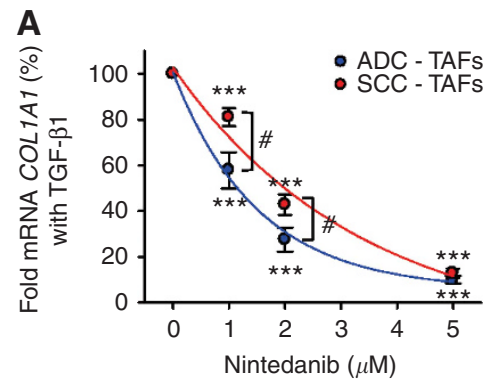

C
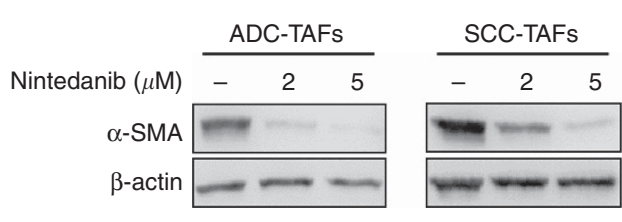

D

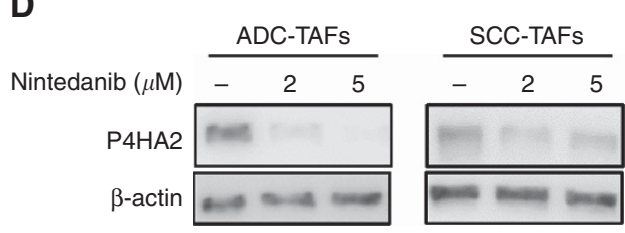

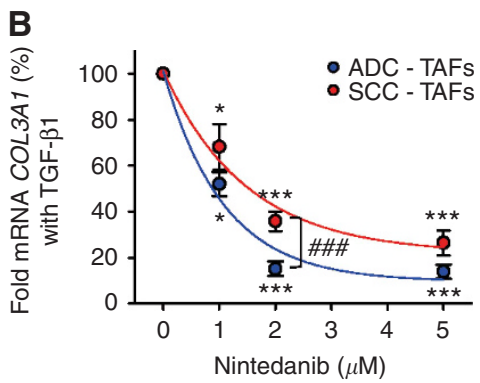
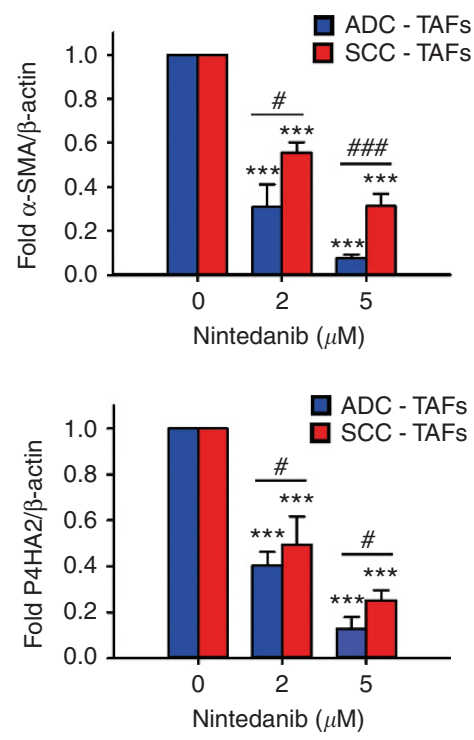

Figure 3. Antifibrotic effects of nintedanib in ADC-TAFs and SCC-TAFs. Antifibrotic effects were assessed in terms of expression of a panel of activation markers including fibrillar collagens COL1A1 ( $\mathbf{A} ; n=6 \mathrm{ADC}, 8 \mathrm{SCC})$ and $\operatorname{COL} 3 \mathrm{~A} 1(\mathbf{B} ; n=6 \mathrm{ADC}, 7 \mathrm{SCC}), \alpha-\mathrm{SMA}(\mathbf{C} ; n=6 \mathrm{ADC}, 6 \mathrm{SCC})$ and P4HA2 ( $;$; $n=5$ ADC, $5 \mathrm{SCC}$ ) upon stimulation with $2.5 \mathrm{ng} \mathrm{ml^{-1 }}$ TGF- $\beta 1$ for 3 days in the presence of increasing nintedanib concentrations as in Figure 2. Continuous lines in ( $\mathbf{A}$ and $\mathbf{B})$ correspond to fittings to an exponential decay function, which were used to assess $I C_{50}$ values shown in

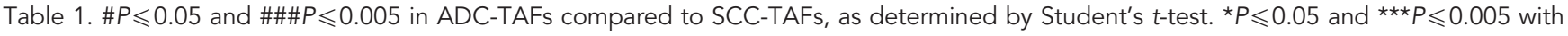
respect to $0 \mu \mathrm{M}$ nintedanib were determined by Student's t-test.
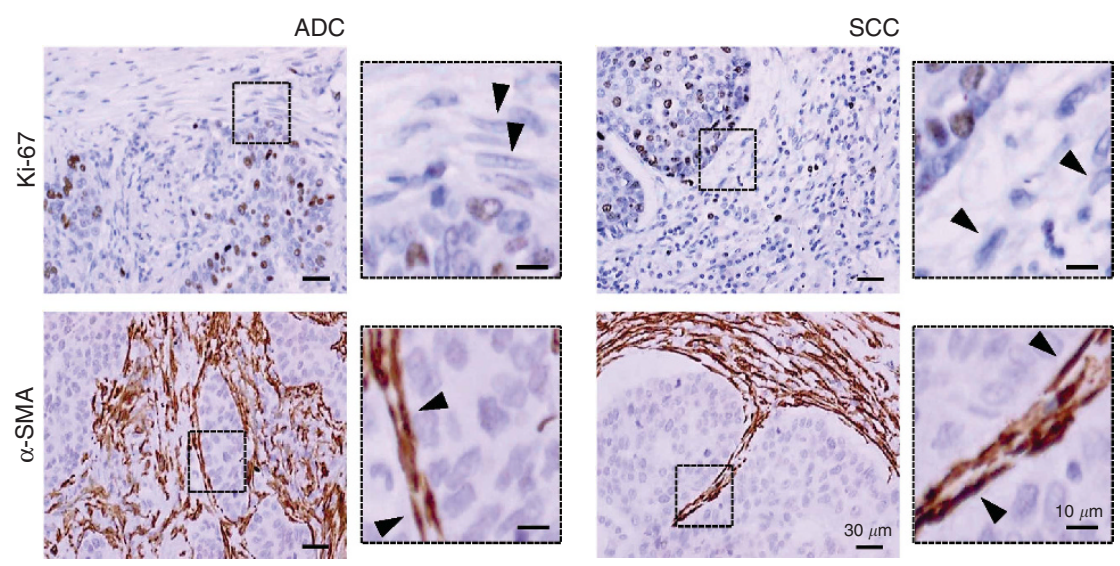

Figure 4. Proliferative and activation status of TAFs in vivo. Illustrative images of Ki-67 and $\alpha$-SMA stromal stainings in histologic sections from ADC and SCC patients obtained with a $\times 40$ objective. Arrowheads point to spindle-shaped nuclei characteristic of fibroblasts. Additional images from other patients are shown in Supplementary Figure S4.

Ki-67 positive (Figure 4 and Supplementary Figure S4). In contrast, virtually all TAFs were positive for the activation marker $\alpha$-SMA in both ADC and SCC (Figure 4), in agreement with previous observations from our group (Puig et al, 2015). Because TAFs are largely quiescent and activated in histologic sections, it is conceivable that the differential antifibrotic effects of nintedanib on ADC-TAFs and SCC-TAFs may be more therapeutically relevant than its antiproliferative function.
Nintedanib abrogates the increase in cancer cell growth and invasion elicited by conditioned medium from activated TAFs in ADC but not SCC. To test the latter interpretation, we assessed the inhibitory impact of nintedanib in two critical pro-tumourigenic effects of activated TAFs: stimulation of growth and invasion of carcinoma cells. For this purpose, we stimulated either ADCTAFs or SCC-TAFs for 3 days with TGF- $\beta 1$ in the presence or absence of $2 \mu \mathrm{M}$ nintedanib, which is comparable to the $I C_{50}$ for 


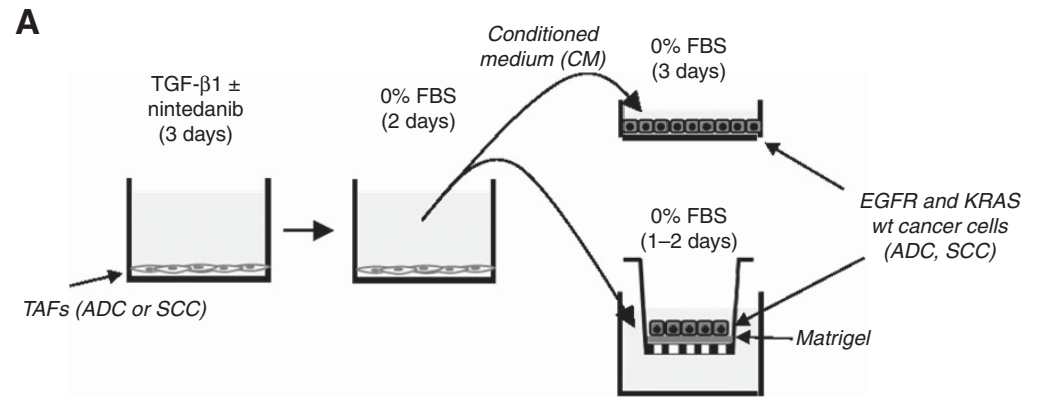

B

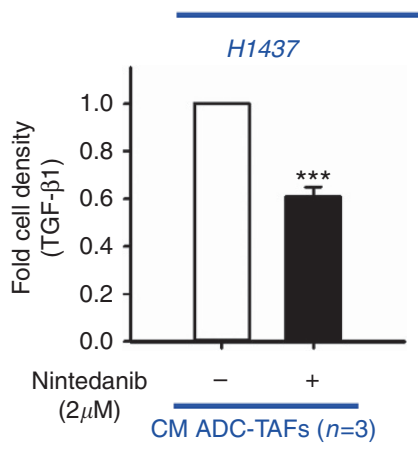

D

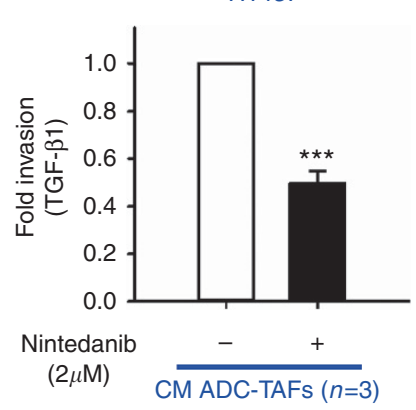

$\mathbf{F}$

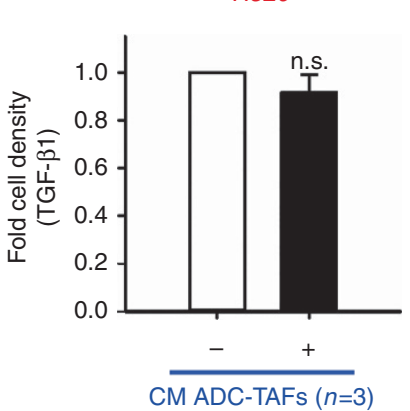

$\mathrm{ADC}$

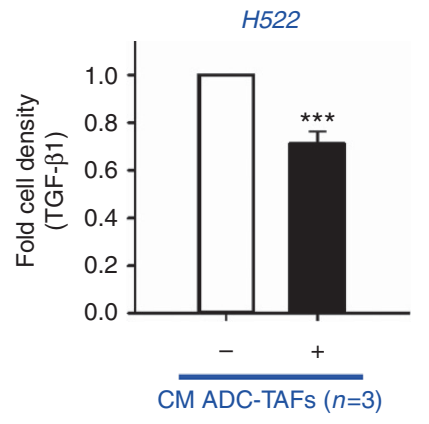

H522

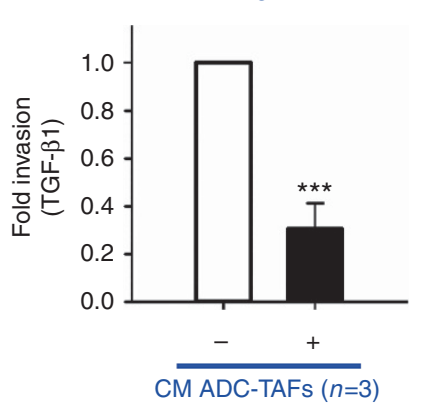

$\operatorname{SCC}$

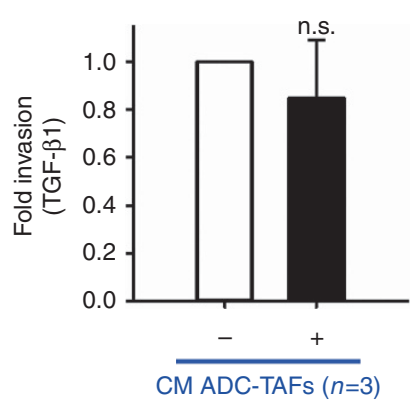

C

scc
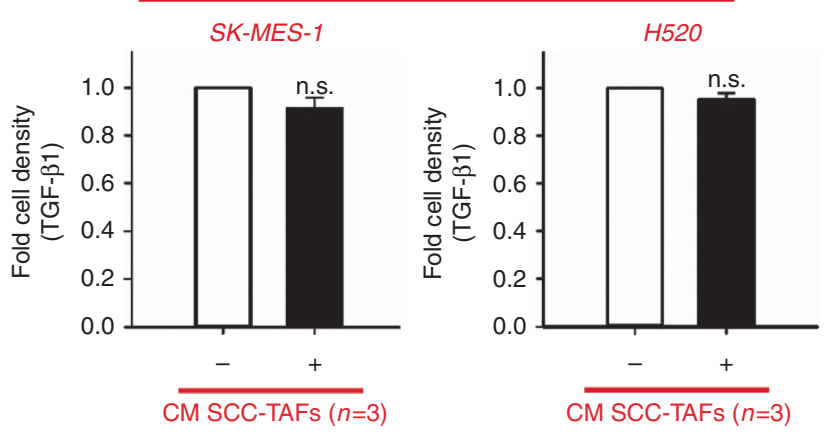

E
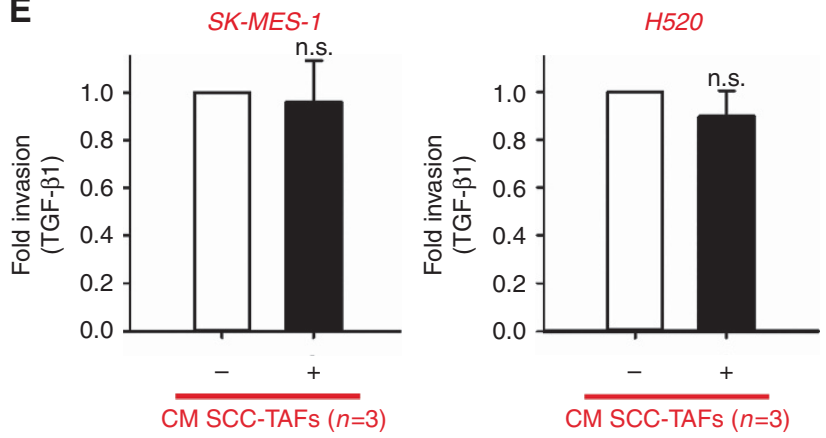

G
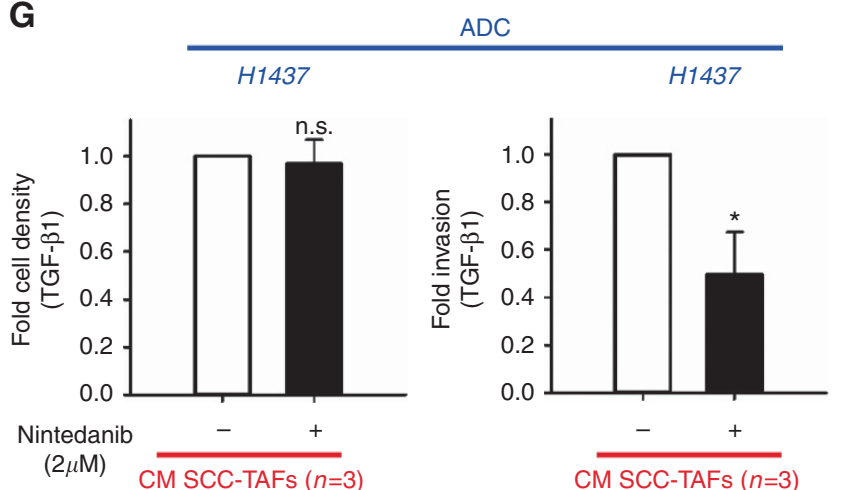

Figure 5. Nintedanib modulation of the tumour-promoting effects of activated TAFs. (A) Outline of the experimental design used to assess the growth (top right) and invasion (bottom right) of carcinoma cell lines stimulated with conditioned medium (CM) from TAFs activated with $2.5 \mathrm{ng} \mathrm{ml}^{-1}$ TGF- $\beta 1$ for 3 days in the presence or absence of $2 \mu \mathrm{m}$ nintedanib and subsequently maintained in serum-free and nintedanib-free medium for 2 days. (B and C) Average fold cell number per field of a panel of wild-type (wt) EGFR and KRAS carcinoma cell lines derived from ADC (H1437, H522) (B) or SCC (SK-MES-1, H520) (C) patients upon stimulation with CM from ADC-TAFs $(n \geqslant 4)$ or SCC-TAFs $(n \geqslant 4)$, respectively, obtained as described in $\mathbf{A}$. (D and $\mathbf{E}$ ) Average fold invasion of the same panel of ADC (D) or SCC (E) carcinoma cell lines induced by different types of CM ( $n \geqslant 4$ for ADC-TAFs and SCC-TAFs) as in (B and $\mathbf{C})$. (F) Growth and invasion of SCC cells (H520) induced by the CM of ADC-TAFs $(n=3)$. (G) Growth and invasion of ADC cells (H1437) induced by the CM of SCC-TAFs $(n=3)$. ${ }^{*} P \leqslant 0.05$ and ${ }^{* \star *} P \leqslant 0.005$ with respect to $0 \mu \mathrm{M}$ nintedanib were determined by Student's $t$-test.

SCC-TAFs (Table 1), washed the medium, replaced it with nintedanib-free and serum-free fibroblast medium, and collected the corresponding $\mathrm{CM}$ as outlined in Figure 5A. In these conditions, no significant changes in fibroblast number were detected regardless of the presence or absence of nintedanib (Supplementary Figure S5). The CM was used to stimulate the 
growth and invasion of a panel of ADC and SCC cell lines that were selected based on their EGFR and KRAS wild-type status to mimic key genetic features of those patients that may be treated with nintedanib (Bronte et al, 2016). As expected, the CM of activated TAFs induced a robust $(\sim 1.5-2$-fold $)$ growth enhancement with respect to serum-free control medium in both ADC and SCC cell lines (Supplementary Figure S5). Remarkably, such growth enhancement of carcinoma cells drop by $\sim 30-40 \%$ when using CM from activated TAFs treated with $2 \mu \mathrm{M}$ nintedanib in ADC cell lines only (Figure 5B), whereas it remained virtually unaltered in SCC cells (Figure 5C). The selective therapeutic effects of nintedanib in ADC cells were even more dramatic when analysing invasion data. Thus, CM of activated TAFs increased markedly ( $\sim 1.5-2.5$-fold) the invasion through Matrigel-coated Transwell inserts in both ADC and SCC cell lines with respect to serum-free control medium (Supplementary Figure S5). In contrast, the observed increase in invasion was reduced by $\sim 60$ $70 \%$ when using CM from activated TAFs treated with $2 \mu \mathrm{M}$ nintedanib in ADC cell lines (Figure 5D), whereas no reduction was observed in SCC (Figure 5E). These in vitro observations are in qualitative agreement with the selective anticancer effects of nintedanib observed in ADC patients in clinical trials (Reck et al, 2014; Novello et al, 2015). Moreover, these results reveal for the first time that nintedanib in vitro induces a robust inhibition of both the growth and invasive advantages provided by soluble factors secreted by activated TAFs in ADC but not SCC.

Finally, to assess the potential contribution of carcinoma cells themselves on the differential therapeutic effects of nintedanib on the carcinoma-TAF cross-talk, we stimulated SCC cells with $\mathrm{CM}$ from ADC-TAFs and vice versa. Four different scenarios could be envisioned. Should the observed differential nintedanib responses be attributed largely to TAFs only, then CM of ADCTAFs treated with nintedanib on SCC cells should induce positive therapeutic responses as in Figure $5 \mathrm{~B}$ and $\mathrm{D}$, whereas the opposite experiment (i.e., CM of SCC-TAFs on ADC cells) should induce negative therapeutic responses as in Figure $5 \mathrm{C}$ and E. Conversely, should the observed responses be attributed largely to carcinoma cells, then the outcomes should be inverted (i.e., CM of ADC-TAFs treated with nintedanib on SCC cells should induce negative therapeutic responses, whereas $\mathrm{CM}$ of SCC-TAFs on ADC cells should induce positive responses). The results are shown in Figure $5 \mathrm{~F}$ and $\mathrm{G}$, where $\mathrm{ADC}$ and SCC samples are labelled in blue and red, respectively, to facilitate the experimental interpretation. $\mathrm{CM}$ of ADC-TAFs treated with nintedanib on SCC cells elicited negative therapeutic responses in terms of both growth and invasion (Figure 5F). In contrast, the CM of SCC-TAFs treated with nintedanib on ADC cells elicited a negative response in terms of growth but a positive response in terms of invasion (Figure 5G). These results support that there is a division of labour between TAFs and carcinoma cells in which both SCC-TAFs and carcinoma cells themselves (ADC and SCC) contribute to the observed differential therapeutic responses to nintedanib.

High nintedanib doses induce vacuolar structures suggestive of autophagy. During the course of our experiments we observed a consistent formation of large vacuolar structures by phase contrast microscopy in both normal fibroblasts and TAFs in all experimental conditions (10\% FBS, TGF- $\beta 1$ ) at the highest nintedanib concentrations examined $(2-5 \mu \mathrm{M})$ (Supplementary Figure S6). Similar vacuolar structures have been recently reported in lung fibroblasts treated with nintedanib elsewhere, and have been interpreted as indicators of autophagy induction (Rangarajan et al, 2016). Autophagy is a highly conserved biological process that allows the degradation and recycling of cellular components. This degradative process has been associated with cellular responses to different types of stress (Schneider and Cuervo, 2014). Accordingly, it is conceivable that high nintedanib concentrations may elicit an autophaghic stress response in both control fibroblasts and TAFs.

\section{DISCUSSION}

Nintedanib is a multi-kinase inhibitor initially designed as a next generation antiangiogenic drug (Hilberg et al, 2008) that has been recently approved to treat patients with advanced lung ADC, based on its selective therapeutic benefits on ADC patients (Reck et al, 2014; McCormack, 2015). However, the biological processes underlying the selective therapeutic functions of this drug on ADC (but not SCC) had remained undefined. We hypothesised that such selective effects could be due, at least in part, to a larger impact of nintedanib on ADC-TAFs compared to SCC-TAFs. To test this hypothesis we used preclinical in vitro models to examine the effects of nintedanib on primary TAFs derived from lung cancer patients and on their tumour-promoting functions on carcinoma cells. To minimise unwanted bias due to sample variability, all TAFs were derived as tissue explants from surgical patients selected upon very stringent criteria. In support to our hypothesis, our results revealed that, in addition to its known antiangiogenic effects (Hilberg et al, 2008; Cenik et al, 2013), nintedanib may be therapeutically effective in ADC owing to its ability to selectively target ADC-TAFs by reducing their activation, which elicits anticancer effects through downregulation of secreted soluble factors in TAFs that stimulate the growth and invasion of carcinoma cells. In agreement with our observations, a significant decrease in activated host fibroblasts was reported in tumour mouse xenografts with ADC cells upon nintedanib treatment, concomitantly with a reduction in tumour size and vessel density (Cenik et al, 2013). The remarkable antifibrotic effects of nintedanib are underscored by our novel observation that this drug was able to markedly reduce the expression of activation markers even in pre-activated fibroblasts. Conversely, our data support that the lack of therapeutic benefit of nintedanib in SCC patients is associated with the poor antifibrotic effect of this drug in SCC-TAFs as well as to its failure to reduce the secretion of factors in TAFs that stimulate the growth and invasion of SCC cells. In addition to the direct contribution of TAFs, our mixed CM experiments support that both ADC and SCC cells contribute themselves to the positive therapeutic effects of nintedanib in ADC but not in SCC, revealing a division of labour between TAFs and carcinoma cells in modulating the subtype-specific nintedanib responses.

Our study underscores the need to analyse the differential secretome of ADC-TAFs and SCC-TAFs to ultimately identify those secreted factors that may provide a pro-growth and pro-invasive soluble microenvironment to ADC cells. Likewise an analysis of the potential differential expression of the corresponding receptors in ADC and SCC cells is warranted to shed light on the mechanisms underlying the cross-talk between TAFs and carcinoma cells in the selective therapeutic effects of nintedanib in ADC. Although identifying these mechanisms was beyond the scope of this study, we can envision potential candidates based on previous studies. Specifically TAF stimulation of carcinoma cell growth has been previously associated with numerous secreted growth factors and cytokines such as HGF, FGF, amphiregulin, CXCL12, TNF- $\alpha$ or Wnt. Likewise, a variety of cytokines, proteases and pro-migratory extracellular matrix components secreted by TAFs have been implicated in the stimulation of carcinoma cell invasion including TGF- $\beta$, CTGF, IL-6, MMPs, TIMPs, tenascin or SPARC (Bhowmick et al, 2004; Mueller and Fusenig, 2004; Bremnes et al, 2011; Ohlund et al, 2014). However defining the precise driving factors awaits future investigations. 
In addition to the secretion of pro-growth and pro-invasive factors, TAFs may contribute indirectly to tumour progression by hindering the diffusion of cancer therapeutics through increased tortuosity and viscosity (Egeblad et al, 2010; Galgoczy et al, 2014), owing to the abundant deposition of fibrillar collagens and other fibrotic extracellular matrix components (Hinz et al, 2012; Jain et al, 2014; Ohlund et al, 2014). Remarkably nintedanib downregulated the mRNA levels of the main fibrillar collagens in fibrosis to a larger extent in ADC-TAFs compared to SCC-TAFs. Likewise nintedanib elicited a larger downregulation of $\mathrm{P} 4 \mathrm{HA} 2$ expression in ADC-TAFs than in SCC-TAFs, which is an essential enzyme for the intracellular stability of the procollagen triple helix that is required for its effective secretion (Chen and Raghunath, 2009; Schwarz, 2015). In agreement with our molecular observations, a recent electron microscopy study reported a downregulation of collagen fibril number and thickness in lung fibroblasts stimulated with TGF- $\beta 1$ in the presence of $1 \mu \mathrm{M}$ nintedanib (Knuppel et al, 2017). Based on these findings, it is conceivable that part of the clinical benefits of the combination of nintedanib with docetaxel in ADC patients are due to the downregulation of fibrillar collagens elicited by nintedanib, for this downregulation may facilitate the delivery of docetaxel to carcinoma cells by removing interstitial barriers that hinder diffusion. In support of this interpretation, we previously reported that the diffusivity of $4 \mathrm{kDa}$ molecules (which is close to the low molecular weight of docetaxel) through type I collagen gels increased by $\sim 20 \%$ upon reducing collagen concentration by $\sim 70 \%$ (Galgoczy et al, 2014), which is a similar reduction to that elicited by $2 \mu \mathrm{m}$ nintedanib on COL1A1 mRNA expression in ADC-TAFs.

ADC-TAFs and SCC-TAFs exhibit distinct phenotypic alterations in terms of growth and integrin signalling in basal conditions (i.e., in the absence of either exogenous pro-growth or proactivation biochemical stimuli) (Puig et al, 2015). In the present study we increased the list of subtype-specific aberrant features in TAFs by reporting differential antifibrotic responses to nintedanib, and revealed similar responses in ADC-TAFs and control fibroblasts. Although the molecular underpinnings underlying all these subtype-specific alterations in TAFs remains to be elucidated, the differential antifibrotic responses to nintedanib strongly suggest that TAF activation in ADC and SCC elicited by TGF- $\beta 1$ may be regulated through distinct mechanisms. In support to this interpretation we recently showed that the TGF- $\beta$ pathway is globally altered in lung TAFs as part of their epigenetic reprogramming with respect to normal fibroblasts (Vizoso et al, 2015). In addition two recent studies have reported the nintedanib inhibition of the activity of TGF- $\beta$ receptor II as well as signalling factors of the TGF- $\beta$ pathway in lung fibroblasts stimulated with TGF- $\beta$ (Hostettler et al, 2014; Rangarajan et al, 2016). Thus, even though the regulatory impact of nintedanib on the TGF- $\beta$ pathway remains incompletely defined (Rangarajan et al, 2016), it is tempting to speculate that the differential antifibrotic effects of nintedanib in ADC-TAFs and SCC-TAFs could be associated with its ability to impact TGF- $\beta$ signalling at different regulatory levels in a subtype-specific fashion.

In addition to antifibrotic functions, nintedanib exhibited robust antiproliferative effects in both normal lung fibroblasts and TAFs when stimulated with $10 \%$ serum, which is rich in PDGF and other fibroblast mitogens (Freshney, 1994). These findings are consistent with previous observations that nintedanib efficiently blocks PDGFR activation and proliferation in normal and fibrotic fibroblasts in vitro and in vivo (Hostettler et al, 2014; Wollin et al, 2014; Huang et al, 2016), and altogether reveal that this drug elicits strong antiproliferative functions on lung fibroblasts in both normal and diseased conditions. However, although such antiproliferative effects appear to be relevant in IPF and other fibrotic disorders, we argue that the therapeutically relevant function of nintedanib against the desmoplastic tumour stroma in ADC is its antifibrotic rather than its antiproliferative effects, because TAFs are largely quiescent and activated in vivo.

The highest nintedanib dose used in our study $(5 \mu \mathrm{M})$ induced the formation of large vacuolar structures in both normal lung fibroblasts and lung TAFs. Similar structures have been recently reported in lung fibroblasts treated with increasing doses of nintedanib up to $2 \mu \mathrm{M}$ elsewhere, and were directly associated with autophagy (Rangarajan et al, 2016). In contrast, it is currently unclear whether nintedanib induces vacuolar structures or autophagy in lung cancer cells (Wollin et al, 2015; Shochet et al, 2016), although autophagy has been reported in different cancer types in response to other tyrosine kinase inhibitors (Han et al, 2011; Rikiishi, 2012). Because previous work has associated autophagy with cell responses to different types of stress (Schneider and Cuervo, 2014), it is conceivable that fibroblast autophagy is a stress response triggered by high nintedanib doses. However, even though the $2-$ $5 \mu \mathrm{M}$ range is larger than the maximum nintedanib concentration reported in plasma $(\sim 1 \mu \mathrm{M})$ during in vivo studies of this drug in tumour xenografts (Hilberg et al, 2008), it remains unknown whether stromal autophagy does occur in patients.

Our observed direct inhibitory effect of nintedanib on the activation and tumour-promoting effects of ADC-TAFs expand previous observations of a direct inhibitory effect of this drug on stromal cell types involved in angiogenesis (Hilberg et al, 2008). Collectively these observations underscore that nintedanib can be considered an anti-stromal drug, for it inhibits multiple tumourpromoting processes by targeting numerous cell types within the tumour stroma. Accordingly, the synergistic therapeutic effects of nintedanib combined with docetaxel reported in ADC patients illustrate an emerging strategy in cancer therapeutics based on combining drugs that target the aberrant tumour stroma with either cytotoxic drugs or targeted drugs against carcinoma cells (Mueller and Fusenig, 2004; Bremnes et al, 2011; Ohlund et al, 2014). However, it should be borne in mind that the latter therapeutic strategy was unsuccessful in SCC patients, supporting that the aberrant carcinoma-TAF cross-talk in SCC is mediated through distinct mechanisms than in ADC. Yet, because our preclinical models of carcinoma-TAF interactions captured the selective therapeutic effects of nintedanib in ADC (but not SCC) reported in clinical trials, we argue that these models may be useful in identifying the mechanisms underlying the poor antifibrotic response of SCC-TAFs to nintedanib.

In summary, our preclinical study reveals that the selective therapeutic benefits of nintedanib combined with docetaxel in $\mathrm{ADC}$ are associated with at least two complementary processes. First, the direct antifibrotic effect of nintedanib on ADC-TAFs, which reduces the deposition of fibrillar collagens, thereby potentially reducing the physical barriers that may hinder the delivery of docetaxel to cancer cells. Second, the reduction of the secretion of yet unidentified soluble factors that stimulate the growth and invasion of ADC cells. Likewise, our results support that the latter therapeutic benefits of nintedanib are largely absent in SCC due to the poor antifibrotic response of SCC-TAFs to this drug. Collectively these results underscore the important role of TAFs in modulating therapy responses.

\section{ACKNOWLEDGEMENTS}

We thank Maeba Polo (UB), Marta Bosch and the Citomics and Biobank Units (IDIBAPS) for technical assistance; Claudi Solà, Ester Pujol and Flavio Solca (Boehringer-Ingelheim) for helpful discussions; and Daniel Navajas and Ramon Farré (UB) for support. This work was further supported by grants from Boehringer-Ingelheim (to JA and NR), the Ministerio de Economía y Competitividad (PI13/02368 and SAF2016-79527-R to JA 
(MINECO/FEDER, UE), PS09/01377 and PI16/00890 to NR), Junta Provincial de Barcelona de l'Associació Espanyola Contra el Càncer AECC (AECC B16-917 to JA), Generalitat de Catalunya AGAUR (SGR 661 to JA), and Ciência sem Fronteiras CNPq (to RI).

\section{CONFLICT OF INTEREST}

This project was partially funded by a research grant with Boehringer-Ingelheim Spain.

\section{REFERENCES}

Abramoff MD, Magelhaes PJ, Ram SJ (2004) Image processing with ImageJ. Biophotonics Int 11(7): 3642.

Awasthi N, Schwarz RE (2015) Profile of nintedanib in the treatment of solid tumors: the evidence to date. Oncotargets Ther 8: 3691-3701.

Bertolini G, D’Amico L, Moro M, Landoni E, Perego P, Miceli R, Gatti L, Andriani F, Wong D, Caserini R, Tortoreto M, Milione M, Ferracini R, Mariani L, Pastorino U, Roato I, Sozzi G, Roz L (2015) Microenvironment-modulated metastatic CD133(+)/CXCR4 $(+) /$ $\operatorname{EpCAM}(-)$ lung cancer-initiating cells sustain tumor dissemination and correlate with poor prognosis. Cancer Res 75(17): 3636-3649.

Bhowmick NA, Neilson EG, Moses HL (2004) Stromal fibroblasts in cancer initiation and progression. Nature 432(7015): 332-337.

Bissell MJ, Hines WC (2012) Why don't we get more cancer? A proposed role of the microenvironment in restraining cancer progression. Nat Med 17(3): 320-329.

Blanco R, Iwakawa R, Tang M, Kohno T, Angulo B, Pio R, Montuenga LM, Minna JD, Yokota J, Sanchez-Cespedes M (2009) A gene-alteration profile of human lung cancer cell lines. Hum Mutat 30(8): 1199-1206.

Bremnes RM, Donnem T, Al-Saad S, Al-Shibli K, Andersen S, Sirera R, Camps C, Marinez I, Busund LT (2011) The role of tumor stroma in cancer progression and prognosis emphasis on carcinoma-associated fibroblasts and non-small cell lung cancer. J Thorac Oncol 6(1): 209-217.

Bronte G, Passiglia F, Galvano A, Barraco N, Listi A, Castiglia M, Rizzo S, Fiorentino E, Bazan V, Russo A (2016) Nintedanib in NSCLC: evidence to date and place in therapy. Therapeut Adv Med Oncol 8(3): 188-197.

Cenik BK, Ostapoff KT, Gerber DE, Brekken RA (2013) BIBF 1120 (Nintedanib), a triple angiokinase inhibitor, induces hypoxia but not EMT and blocks progression of preclinical models of lung and pancreatic cancer. Mol Cancer Therapeut 12(6): 992-1001.

Chen CZ, Raghunath M (2009) Focus on collagen: in vitro systems to study fibrogenesis and antifibrosis state of the art. Fibrogenesis Tissue Repair 2: 7-7.

Chen Z, Fillmore CM, Hammerman PS, Kim CF, Wong K-K (2014) Nonsmall-cell lung cancers: a heterogeneous set of diseases. Nat Rev Cancer 14(8): 535-546.

Egeblad M, Rasch MG, Weaver VM (2010) Dynamic interplay between the collagen scaffold and tumor evolution. Curr Opin Cell Biol 22(5): 697-706.

Ferlay J, Steliarova-Foucher E, Lortet-Tieulent J, Rosso S, Coebergh JWW, Comber H, Forman D, Bray F (2013) Cancer incidence and mortality patterns in Europe: estimates for 40 countries in 2012. Eur J Cancer 49(6): 1374-1403.

Freshney RI (1994) Culture of animal cells. A manual of basic technique. 3rd edn. Wiley-Liss.

Galgoczy R, Pastor I, Colom A, Gimenez A, Mas F, Alcaraz J (2014) A spectrophotometer-based diffusivity assay reveals that diffusion hindrance of small molecules in extracellular matrix gels used in 3D cultures is dominated by viscous effects. Colloid Surf B-Biointerfaces 120: 200-207.

Han WD, Pan HM, Chen Y, Sun J, Wang YS, Li J, Ge WT, Feng LF, Lin XY, Wang XJ, Wang X, Jin HC (2011) EGFR tyrosine kinase inhibitors activate autophagy as a cytoprotective response in human lung cancer cells. Plos One 6(6): 8.

Hanahan D, Weinberg RA (2011) Hallmarks of cancer: the next generation. Cell 144(5): 646-674.

Hilberg F, Roth GJ, Krssak M, Kautschitsch S, Sommergruber W, Tontsch-Grunt U, Garin-Chesa P, Bader G, Zoephel A, Quant J, Heckel A,
Rettig WJ (2008) BIBF 1120: triple angiokinase inhibitor with sustained receptor blockade and good antitumor efficacy. Cancer Res 68(12): $4774-4782$.

Hinz B, Phan SH, Thannickal VJ, Prunotto M, Desmoulière A, Varga J, De Wever O, Mareel M, Gabbiani G (2012) Recent developments in myofibroblast biology. Paradigms for connective tissue remodeling. Am J Pathol 180: 1340-1356.

Hostettler KE, Zhong J, Papakonstantinou E, Karakiulakis G, Tamm M, Seidel P, Sun Q, Mandal J, Lardinois D, Lambers C, Roth M (2014) Anti-fibrotic effects of nintedanib in lung fibroblasts derived from patients with idiopathic pulmonary fibrosis. Respir Res 15: 157.

Huang J, Beyer C, Palumbo-Zerr K, Zhang Y, Ramming A, Distler A, Gelse K, Distler O, Schett G, Wollin L, Distler J (2016) Nintedanib inhibits fibroblast activation and ameliorates fibrosis in preclinical models of systemic sclerosis. Ann Rheum Dis 75: 883-890.

Jain RK, Martin JD, Stylianopoulos T (2014) The role of mechanical forces in tumor growth and therapy. Annu Rev Biomed Eng 16: 321-346.

Kalluri R, Zeisberg M (2006) Fibroblasts in cancer. Nat Rev Cancer 6(5): 392-401.

King Jr TE, Pardo A, Selman M (2011) Idiopathic pulmonary fibrosis. Lancet 378(9807): 1949-1961.

Knuppel L, Ishikawa Y, Aichler M, Heinzelmann K, Hatz R, Behr J, Walch A, Bachinger HP, Eickelberg O, Staab-Weijnitz CA (2017) A novel antifibrotic mechanism of nintedanib and pirfenidone: inhibition of collagen fibril assembly. Am J Respir Cell Mol Biol 57(1): 77-90.

Koslowski R, Seidel D, Kuhlisch E, Knoch KP (2003) Evidence for the involvement of TGF-beta and PDGF in the regulation of prolyl 4-hydroxylase and lysyloxidase in cultured rat lung fibroblasts. Exp Toxicol Pathol 55(4): 257-264.

Labernadie A, Kato T, Brugués A, Serra-Picamal X, Derzsi S, Arwert E, Weston A, González-Tarragó V, Elosegui-Artola A, Albertazzi L, Alcaraz J, Roca-Cusachs P, Sahai E, Trepat X (2017) A mechanically active heterotypic E-cadherin/N-cadherin adhesion enables fibroblasts to drive cancer cell invasion. Nat Cell Biol 19: 224-238.

Livak KJ, Schmittgen TD (2001) Analysis of relative gene expression data using real-time quantitative PCR and the $2(-$ Delta Delta $\mathrm{C}(\mathrm{T}))$ Method. Methods 25(4): 402-408.

Lugo R, Gabasa M, Andriani F, Puig F, Facchinetti F, Ramírez J, Gómez-Caro A, Pastorino U, Fuster G, Almendros I, Gascón P, Davalos A, Reguart N, Roz L, Alcaraz J (2016) Heterotypic paracrine signaling drives fibroblast senescence and tumor progression of large cell carcinoma of the lung. Oncotarget 7: 82324-82337.

McCormack PL (2015) Nintedanib: first global approval. Drugs 75(1): 129-139.

Mesner PW, Winters TR, Green SH (1992) Nerve growth-factor withdrawalinduced cell-death in neuronal pc12 cells resembles that in sympathetic neurons. J Cell Biol 119(6): 1669-1680.

Mueller MM, Fusenig NE (2004) Friends or foes - Bipolar effects of the tumour stroma in cancer. Nat Rev Cancer 4(11): 839-849.

Navab R, Strumpf D, Bandarchi B, Zhu CQ, Pintilie M, Ramnarine VR, Ibrahimov E, Radulovich N, Leung L, Barczyk M, Panchal D, To C, Yun JJ, Der S, Shepherd FA, Jurisica I, Tsao MS (2011) Prognostic geneexpression signature of carcinoma-associated fibroblasts in non-small cell lung cancer. Proc Natl Acad Sci USA 108(17): 7160-7165.

Novello S, Kaiser R, Mellemgaard A, Douillard J-Y, Orlov S, Krzakowski M, von Pawel J, Gottfried M, Bondarenko I, Liao M, Barrueco J, GaschlerMarkefski B, Griebsch I, Palmer M, Reck M. Grp LU-LS (2015) Analysis of patient-reported outcomes from the LUME-Lung 1 trial: a randomised, double-blind, placebo-controlled, Phase III study of second-line nintedanib in patients with advanced non-small cell lung cancer. Eur J Cancer 51(3): 317-326.

Ohlund D, Elyada E, Tuveson D (2014) Fibroblast heterogeneity in the cancer wound. J Exp Med 211(8): 1503-1523.

Puig M, Lugo R, Gabasa M, Gimenez A, Velasquez A, Galgoczy R, Ramirez J, Gomez-Caro A, Busnadiego O, Rodriguez-Pascual F, Gascon P, Reguart N, Alcaraz J (2015) Matrix stiffening and beta(1) integrin drive subtype-specific fibroblast accumulation in lung cancer. Mol Cancer Res 13(1): 161-173.

Rangarajan S, Kurundkar A, Kurundkar D, Bernard K, Sanders YY, Ding Q, Antony VB, Zhang J, Zmijewski J, Thannickal VJ (2016) Novel mechanisms for the antifibrotic action of nintedanib. Am J Respir Cell Mol Biol 54(1): 51-59.

Reck M, Kaiser R, Mellemgaard A, Douillard J-Y, Orlov S, Krzakowski M, von Pawel J, Gottfried M, Bondarenko I, Liao M, Gann C-N, Barrueco J, Gaschler-Markefski B, Novello S, Grp LU-LS (2014) Docetaxel plus 
nintedanib versus docetaxel plus placebo in patients with previously treated non-small-cell lung cancer (LUME-Lung 1): a phase 3, doubleblind, randomised controlled trial. Lancet Oncol 15(2): 143-155.

Richeldi L, du Bois RM, Raghu G, Azuma A, Brown KK, Costabel U, Cottin V, Flaherty KR, Hansell DM, Inoue Y, Kim DS, Kolb M, Nicholson AG, Noble PW, Selman M, Taniguchi H, Brun M, Le Maulf F, Girard M, Stowasser S, Schlenker-Herceg R, Disse B, Collard HR, Investigators IT (2014) Efficacy and safety of nintedanib in idiopathic pulmonary fibrosis. N Engl J Med 370(22): 2071-2082.

Rikiishi H (2012) Autophagic action of new targeting agents in head and neck oncology. Cancer Biol Ther 13(11): 978-991.

Schneider JL, Cuervo AM (2014) Autophagy and human disease: emerging themes. Curr Opin Genet Dev 26: 16-23.

Schwarz RI (2015) Collagen I and the fibroblast: high protein expression requires a new paradigm of post-transcriptional, feedback regulation. Biochem Biophys Rep 3: 38-44.

Shochet GE, Israeli-Shani L, Koslow M, Shitrit D (2016) Nintedanib (BIBF 1120) blocks the tumor promoting signals of lung fibroblast soluble microenvironment. Lung Cancer 96: 7-14.

Taylor JR (1997) An Introduction to Error Analysis. University Science Books: Sausalito.
Vizoso M, Puig M, Carmona FJ, Maqueda M, Velásquez A, Gómez A, Labernadie A, Lugo R, Gabasa M, Rigat de Brugarolas LG, Trepat X, Ramírez J, Reguart N, Morán S, Vidal E, Perera A, Esteller M, Alcaraz J (2015) Aberrant DNA methylation in non small cell lung cancer associated fibroblasts. Carcinogenesis 36: 1453-1463.

Wollin L, Maillet I, Quesniaux V, Holweg A, Ryffel B (2014) Antifibrotic and anti-inflammatory activity of the tyrosine kinase inhibitor nintedanib in experimental models of lung fibrosis. J Pharmacol Exp Therapeut 349(2): 209-220.

Wollin L, Wex E, Pautsch A, Schnapp G, Hostettler KE, Stowasser S, Kolb M (2015) Mode of action of nintedanib in the treatment of idiopathic pulmonary fibrosis. Eur Respir J 45(5): 1434-1445.

(c) (1) (2) (2) This work is licensed under the Creative Commons c. ${ }_{\text {BY }}$ NC SA Attribution-Non-Commercial-Share Alike 4.0 International License. To view a copy of this license, visit http:// creativecommons.org/licenses/by-nc-sa/4.0/

(C) The Author(s) named above 2017

Supplementary Information accompanies this paper on British Journal of Cancer website (http://www.nature.com/bjc) 